\title{
Translator's Adaptation and Selection on TCM Textbook Translation for International Students
}

\author{
Yu-shan Zhao ${ }^{1}$, Dan Shao ${ }^{2}$, Zhao-hui Liu ${ }^{3}$ \\ 1) Department of Foreign Languages, North China Electric Power University, Changping, Beijing, China \\ ${ }^{2)}$ Department of Foreign Languages, North China Electric Power University, Changping, Beijing, China \\ ${ }^{3)}$ Department of Foreign Languages, North China Electric Power University, Changping, Beijing, China
}

\begin{abstract}
From the perspective of Eco-translatology, this paper analyzes the translational eco-environment of TCM textbook for international students, putting forward its complexity and specialty. With the application of "Translation as Adaptation and Selection" theory, this paper further proposes that in translation of TCM textbook for international students, translator-centeredness should be ensured by a translator's selective adaptation and adaptive selection, in order to fit to its translational eco-environment and choose suitable target texts, thus to keep the eco-balance.
\end{abstract}

Keywords - international students, bilingual TCM textbooks, translational eco-environment, translator-centeredness, adaptation and selection

\section{留学生中医双语教材翻译的译者适应与选择 以供来华留学生用《中医基础理论》（第 2 版）为例 赵玉闪 邵丹 刘朝晖 \\ 华北电力大学外国语学院, 昌平, 北京, 中国}

摘 要 本文从生态翻译学的视角出发, 对留学生中医双语教材的翻译生态环境进行分析, 指出其复杂性和特殊性。通过引用译者适应 /选择理论, 进一步阐释译者在进行留学生中医双语教材翻译时, 应保持译者中心地位, 进行选择性适应和适应性选择, 即适应其翻译 生态环境并选择合适译文, 以达到生态平衡的目的。

关键词 留学生, 中医双语教材, 翻译生态环境, 译者中心, 适应和选择

1. 前言

随着国内外中医研究热潮的出现, 中医对外教学不断 发展并广泛流行, 越来越多的海外学生到中国学习中医知 识, 留学生中医双语教材应运而生。这不仅对教材的中文 编写提出了更高的要求, 更应引起人们对译文的重视。然 而, 受诸多因素制约, 留学生中医双语教材的英译版本质 量参差不齐。同时, 缺乏理论层面的指导和规范是目前国 内中医双语教材英译面临的主要问题。

人民卫生出版社在 2007 年发行了一套（共 10 册）全 国高等中医药院校汉英双语教材·供来华留学生用, 此套教
材翻译队伍大、译者素质高、译文格式和内容规范, 是中 医双语教材英译实践的一大突破。本文以《中医基础理论》

(第 2 版) (以下称《理论》) 一书为范例, 从生态翻译学 视角出发, 对留学生中医双语教材英译进行研究, 阐明在 翻译过程中, 译者是如何适应和选择的。

\section{2. 留学生中医双语教材的翻译生态环境}

生态翻译学是一种生态视域下的翻译学研究, 注重生 态平衡, 寻求译者、文本及译境三者相互间的和谐。其中“译 境”即为翻译生态环境, 指的是“原文、原语和译语所呈现

华北电力大学教改项目（2014JG69）; 中央高校基本科研业务费专项资金资助（华北电力大学，项目号：2014ZD25） 
的“世界”，是语言、交际、文化、社会，以及作者、读者、 委托者等互联互动的整体, 是制约译者最佳适应和优化选 择的多种因素的集合”[1]。对于留学生中医双语教材的英 译, 要寻求其和谐与平衡, 全面把握留学生中医双语教材 的翻译生态环境尤为重要。

翻译生态环境可分为主体环境和客体环境。主体翻译 生态环境包括“译者、作者、读者、出版商、洽谈商、审稿 人等”; 客体翻译生态环境包括“原文本、译本、文体功能、 翻译策略、翻译规约等” [1]。从内容上看, 主体翻译生态环 境的构成等同于翻译群落, “指的是与特定翻译活动的发 生、发展、操作、结果、功能、效果等彼此影响相互作用 的、与翻译活动相关的“诸者”的集合体”[1]。在留学生中医 双语教材的翻译中, 翻译生态环境主要有译者、编者、委 托者、读者、原文、社会、文化等因素, 从主客体翻译生 态环境的角度来看, 留学生中医双语教材翻译生态环境具 有其复杂性和特殊性。

\section{1 主体翻译生态环境的复杂性和特殊性}

中医教材主体翻译生态环境的特殊性和复杂性主要体 现在译者素质要求特殊性, 中文内容编写群体的特殊性, 出版商的要求具有特殊性及读者群体的特殊性。

首先, 中医教材翻译对译者素质具有特殊要求。在翻 译过程中, 译者是连接作者和读者、源语文本和译文文本 的中间角色, 是重要的桥梁和纽带。因此, 译者需具备如 下素质: 深入理解和灵活运用汉英两种语言的能力, 特别 是语古汉语和中医专业术语; 中医专业背景知识; 汉英不 同文化的把握。对于整个教材翻译而言, 采取多译者合作 翻译情况较为普遍, 这也需要各译者之间分工协作、细致 认真。以上素质具备其一尚且困难, 中医教材翻译译者需 兼备所有, 因此, 对译者而言是一个挑战。

其次, 中文内容编写者, 即编者群体特殊。一套标准、 完善的中医教材的出炉, 需要经过编委会成员共同努力。 以 2007 年人卫社出版的汉英双语教材为例, 其编译委员会 成员都来自于国内各中医药院校, 具有深厚的中医药背景 知识和教学及理论研究经验。这些因素对于留学生中医教 材内容的精准和规范起到了积极的保障作用。

再次, 委托者, 即出版商要求特殊。委托者的要求代 表市场的要求, 是综合经济、社会等多方面因素而提出的。 留学生中医教材面向国内高等中医药院校发行, 出版商既 要寻求潜在的消费者, 注重经济利益, 同时又要提高出版 物的实用性、可读性。因此翻译时要充分考虑出版商提出 的条件, 以满足其针对留学生中医双语教材英译的要求。

最后, 也是最重要的因素, 读者, 即来华学习中医知
识的留学生和对外中医教师, 群体及需求特殊。留学生中 医教材的读者分为两类: 在华高等中医药院校攻读学位的 外国留学生; 是进行中医对外教学的中医教师。

就留学生情况来说, 根据国家中医药管理局公布的数 据[2]（表 1)，2008-2012 年招收的来华学习中医留学生数 都在 1400 人以上, 且留学生分别来自不同的大洲, 但明显 集中在亚洲地区。可见, 留学生人数已基本稳定, 这说明 中医在国际上的地位已经呈稳固发展态势。同时, 中医专 业留学生来自不同的大洲, 但招收的亚洲留学生占总招生 人数的比率维持在 $70 \%$ 左右, 这说明中医在世界范围内都 受到关注, 在亚洲尤为流行。由于留学生来自不同的国家 和地区, 文化背景和生活习俗各异。并且, 不同留学生的 留学目的各异, 专业知识背景和汉语程度也不尽相同。这 些因素中, 语言因素是留学生学习中医知识的第一大障碍。 即便中医专业对留学生汉语要求高于其他学科, “现行的汉 语考试要求测试成绩达到 6 级以上的留学生才有资格申请 中医专业的学历教育” [3], 但对于母语为汉语的中国人学习 起来都无法顺利把握的中医语言, 特别是古汉语和中医专 业术语, 留学生“必然感到专业词汇上的困难较大”[4]。因 此, 中医教材英译的质量对留学生的学习效果影响甚大。

中医双语教材的另一类读者, (对外中医教师) 对中医 教材英译版本的需求程度也很大。对外中医教师在进行授 课的时候, 为了能让留学生更好的理解教材内容, 既需要 用通俗的汉语讲解课本内容, 有时也会用到英语, 即中英 文穿插授课。这样, 中医教材英译版本就成了对外中医教 师的重要参考依据。

表 1 2008-2012 年来华攻读中医学位的留学人员分布情况

\begin{tabular}{|c|c|c|c|c|c|}
\hline $\begin{array}{c}\text { 年份 } \\
\text { 招生数 }\end{array}$ & 2012 & 2011 & 2010 & 2009 & 2008 \\
\hline 洲别 & & & & & \\
\hline 亚 洲 & 1,234 & 1,279 & 1,374 & 1,261 & 1,368 \\
\hline 非 洲 & 48 & 52 & 31 & 18 & 10 \\
\hline 欧 洲 & 111 & 195 & 119 & 71 & 158 \\
\hline 北美洲 & 133 & 211 & 54 & 71 & 116 \\
\hline 南美洲 & 51 & 87 & 15 & 15 & 11 \\
\hline 大洋洲 & 13 & N/A & 45 & N/A & 30 \\
\hline 澳 洲 & N/A & 33 & N/A & 29 & N/A \\
\hline 总 计 & 1,590 & 1,857 & 1,638 & 1,465 & 1,693 \\
\hline
\end{tabular}

\section{2 客体翻译生态环境的复杂性和特殊性}

中医教材客体翻译生态环境的特殊性和复杂性主要体 现在中医教材语言、风格及内容复杂且特殊, 社会和人文 
因复杂且特殊。

中医教材, 即原文, 其语言、风格、内容复杂且特殊。 从语言层面来看, 中医具有悠久的历史, 其理论体系的形 成可以追溯到战国至秦汉时期, 因此现有很多中医著作都 沿袭了中医典籍的语言特色, 中医教材自然也不例外。如, 使用古汉语, 术语多为三字格、四字格, 如“气血两虚”, “母 病及子”。从风格层面来看, 教材属于传播知识的载体, 重 解释说明。同时, 中医学根植于中国传统哲学与文化之中, 中医教材兼具文学哲学性和科技性, 其风格既包含文哲类 作品的可读性和哲理性, 又含有科技类作品的逻辑性和严 谨性。从内容层面来看, 中医教材的编写针对来华留学生 的学习特点及教学需要, 对内容进行适当处理, 重视基础、 强调实用、简明扼要。综合以上语言、风格和内容三个层 面, 留学生中医教材的原文语言复杂、风格特殊、内容针 对性强, 这就对其英译提出了更高的要求。

此外, 中医教材的客体翻译生态环境中, 社会和人文 因素对译者的翻译影响颇深。社会因素主要包括: 中医的 国内国际地位不断上升并趋于稳定, 引来了诸多来华学习 中医知识的留学生, 由此产生了对中医双语教材的需求; 中医热潮引发了出版市场的中医倾向性, 出版商为寻求利 益最大化纷纷将目光投向采手可热的中医, 然而过分注重 经济利益导致中医双语教材缺乏统一的标准, 且质量参差 不齐, 这使读者面对众多版本的双语教材无所是从。人文 因素方面涉及一文化背景的不同使各国留学生思维方式 各异, 特别是中西方思维模式存在显著差异, 这导致留学 生在理解体现中国传统哲学文化的中医上困难加倍。

不论是中医教材的主体翻译生态环境还是客体翻译生 态环境, 都对中医教材的英译影响甚大。因此对翻译生态 环境的充分了解可以帮助译者与翻译生态环境达到和谐统 一, 大大提高翻译质量。

\section{3. 留学生中医教材翻译中译者的适应与选择}

生态翻译学赋予了译者中心地位, 即“突出译者在翻译 过程中的这种中心地位和主导作用”[5]。同时, 生态翻译学 的适应选择理论印证了译者的中心地位一译者在翻译过程 中要进行适应性选择和选择性适应。需要适应的是翻译生 态环境, 需要选择的是合适的译文。

想要进行优质的留学生中医教材翻译, 面对如此纷繁 复杂的翻译生态环境, 译者中心地位的保障和译者的适应 与选择尤为重要。那么, 在留学生中医教材翻译的时候, 译者的中心地位是如何体现的, 译者又应该进行怎样的适 应和选择呢? 本文试图以《中医基础理论》(第 2 版) 为例, 阐释如上问题。
3.1 译者的中心地位

从整体来看, 《理论》的编写和翻译工作均由教材编译 委员会分工完成, 其中主编柴可夫统审中文稿部分, 主译 张庆荣统审英文稿部分。如果说译者在翻译中处于中心地 位, 那么主译则是中心的中心, 其在选择成员组建整个译 者队伍、统领并进行合理分工、规范全书翻译标准、统一 整体语言风格, 乃至整个翻译过程中始终起着重要的作用。 但是要保障译者的中心地位, 仅仅依靠主译一个人的力量 是不够的, 要提高翻译队伍整体素质。因此, 就整个译者 团队成员的组成来看, 每个译者都要拥有相当高的素质: 既要有相当的中医药专业背景, 又要有准确把握汉英两种 语言和文化的能力, 特别是对古汉语和中国古代哲学思想 的理解能力。根据《理论》编译委员会名单, 可知其委员 均为中医药院校出身的学者, 这就满足了翻译者具备中医 专业知识这一要求。并且, 目前中医界对中医英语的重视 程度明显提高, 各类中医英语教材、中医汉英双语词典等 的出版为培养合格的中医英译译者做出了突出的贡献。与 此同时, 优质的译文也倚仗整个翻译团队的齐心协力、精 益求精。只有在译者具备应有的素质、完备的团队和领导, 以及成员间团结一致、不断精进的条件下, 译者的中心地 位才能很好地体现和得到保障。

\section{2 译者的选择性适应与适应性选择}

生态翻译学的前身是翻译适应选择论, 是由达尔文“适 应选择”这一学说发展而来, 因而, 在生态翻译学发展的过 程中, 适应选择论成为其中一个重要的基础性理论。翻译 适应选择论认为, “翻译是译者适应翻译生态环境而对文本 进行移植的选择活动”[1]。“对于生存, 适应是必须的; 对 于进化 (发展), 选择是不可或缺的。”[1]适应和选择二者 水乳交融, 联系紧密, 适应要选择性适应, 选择要适应性 选择。在中医教材翻译中, 译者要能够适应其主客体翻译 生态环境, 并在适应的基础上选择合适的译文。

对于中医教材中译者这一主体翻译生态环境而言, 其 最重要的一点就是要在复杂的翻译生态环境中坚持自我、 完善自身素质以具备中医教材翻译能力, 选择正面及有实 际意义的环境去适应, 拒绝与那些只注重经济利益的环境 同流合污。做到认真保质翻译, 不浮躁。

同时, 译者在坚持自我中, 要适应译者以外翻译群落 的要求, 需综合考虑编者的意图、委托者的要求及读者的 需求, 进行有选择的适应。不论是教材中文内容编者还是 委托者, 其目的都是为了将中医理论知识传递给读者, 即 面向留学生和对外中医教师。从这一点来看, 译者在进行 留学生中医教材英译的时候, 充分考虑留学生和对外中医 
教师的需求和特点尤为重要。如上文提到那样, 来华学习 中医的留学生来自各个大洲, 但主要来自亚洲, 他们不仅 汉语基础不一, 英语基础也同样各异。为满足各国学生的 普遍需求, 译者在保证译文用语地道的同时, 应该选择使 用通俗易懂的语言来讲解中医知识, 尤其是那些晦涩难懂 的中医术语和古汉语成分。例如:

原文: 脾虚病人, 面见青色, 为木虚土乘; 心气虚弱 者, 面见黑色, 为火虚水乘。

译文: A patient with spleen deficiency accompanied with a greenish complexion implies that wood (Liver) subjugates earth (spleen) with deficiency. A patient with heart disease with darkish complexion suggests that water (kidney) subjugates fire (heart) with deficiency.

上例原文中“木虚土乘”、“火虚水乘”在母语为汉语的中 国人看来都未必能摸清其中含义, 更不用说汉语是第二语 言的留学生了。为了既保证原文信息不丢失, 又能将原文 所指之意真正体现, 译者在这里很巧妙地运用括号加注进 行解释, 将原本抽象的“木”、“土”、“火”、“水”按原意译出 的同时，分别运用括号注明其所代表的“肝”、“脾”、“肾”、 “心”。这种方法不仅简洁而且达意, 可谓很好地做出了适 应和选择。

客体翻译生态环境方面, 译者要适应原文医哲相融这 一内容特色, 充分了解不同地区间的文化差异, 选择合适 的表达方式来让各国留学生理解文字背后所蕴含的中国传 统文化。尤其是中医中体现中国传统哲学的部分多用古汉 语词汇, 更加大了翻译的难度。例如:

原文: 协调阴阳, 补偏救弊, 恢复其相对平衡的状态, 促进其阴平阳秘, 乃是临床治疗的根本法则之一。

译文: Coordinating yin and yang to remedy and restore their relative balance is one of the cardinal principles in clinical treatment.

上例中，原文提及“补偏救弊”、“阴平阳秘”，体现了中 国传统哲学中阴阳平衡的观点, 然而这些词汇使留学生理 解上困难重重。而整个原文要阐述的意思便是“协调阴阳使 其恢复平衡是临床治疗的根本法则之一。”因此, 译者在翻 译中选择将文化词“补偏救弊”、“阴平阳秘”直接融在整个句 子中, 将其代表的“阴阳平衡”翻译出来, 在正确体现原意 的同时, 免去了文化词所能带来的理解误区。

此外, 由于中医双语教材是为了传递中医知识给留学 生, 其行文风格上的最大特点就是重解释, 出现很多定义
类语句。因此, 译者在翻译的时候, 要选择保留原文的这 一风格, 解释为主, 对于定义类语句通常进行直译。例如:

原文: 津液是体内一切正常水液的总称。

译文: Body fluid is a general term for all normal liquids in the body.

像上例这样的定义类语句在《理论》中非常常见, 而 翻译的时候书中基本采取直译的方式进行。原文在下定义 的时候即采取解释性语言, 风格简单明了, 利于读者去理 解, 因此, 在进行翻译的时候, 要保持相同风格, 着重解 释, 选择直译。

\section{4. 结论}

作为留学生学习中医知识的重要辅助材料, 留学生中 医双语教材的英文版本质量好坏影响甚大。为了能够提高 教材翻译质量, 形成规范, 运用生态翻译学译者的适应选 择理论来指导翻译有其积极意义。通过对留学生中医教材 内外翻译生态环境的剖析, 可以让译者更好地适应翻译生 态环境和选择译文, 从而真正体现译者的中心地位。其实, 在进行中医教材翻译的时候, 译者会不断适应翻译生态环 境和选择译文, 远远多于本文列举的几种情况。由于篇幅 有限、笔者研究尚未全面, 笔者仅在本文举出突出情形, 抛砖引玉, 希望今后能有更多有关留学生中医教材翻译的 研究, 以丰富翻译理论层面对于中医教材翻译的指导。

\section{参考文献(References)}

[1] G.S. Hu, Eco-translatology-Construction and Interpretation. Beijing: The Commercial Press, 2013.

[2] http://www.satcm.gov.cn.

[3] Y. Huang, "On Current Chinese Language Level and Education of International Traditional Chinese Medical Students," Journal of Chengdu University of TCM(Education Science Edition), vol. 13, no. 3, pp. 29-30, 2011.

[4] Y.S. Zhou and Y. Jin, "On Current Chinese Language Level of International Traditional Chinese Medical Students," Southern Medical Education, no. 4, pp. 19-20, 2009.

[5] G.S. Hu, "From 'Translator's Subjectivity' to 'Translator -centeredness'," Chinese Translators Journal, vol. 25, no. 3, pp. 10-16, 2004. 